\title{
Recurrent pancreatitis in a patient with primary hyperparathyroidism (PHPT)
}

\author{
S Pathmanthan ${ }^{1}$, D U S Bulugahapitiya ${ }^{2}$, Bavantha Gamage ${ }^{3}$ \\ Sri Lanka Journal of Diabetes, Endocrinology and Metabolism 2013; 3: 35-38
}

\begin{abstract}
Primary hyperparathyroidism (PHPT) is an uncommon cause of pancreatitis. Both acute and chronic pancreatitis has been reported in association with primary hyperparathyroidism. However the cause and effect relationship between the two still evokes controversy. We report a case of a 53 year old woman who presented with recurrent episodes of pancreatitis. Serum calcium during one of the attack was normal but repeat calcium estimation showed raised serum calcium. Eventually, the finding of an elevated intact PTH level led to preoperative localization of a parathyroid adenoma. A solitary parathyroid adenoma was found during the open neck exploration. Post-surgery, serum calcium returned to normal and there was no recurrence of the abdominal symptoms. Our paper also reviews the literature on pancreatitis and PHPT and supports our belief that pancreatitis is one of the symptoms of PHPT and it would be prudent to check serum calcium in all patients presenting with unexplained pancreatic disease.
\end{abstract}

Key words: primary hyperparathyroidism, pancreatitis, parathyroid adenoma

\section{Introduction}

Primary hyperparathyroidism (PHPT) is an endocrine disorder characterized by excessive secretion of parathyroid hormone from one or more parathyroid glands. The clinical profile of PHPT in the western countries has shifted from a symptomatic disorder to a more asymptomatic state. However symptomatic PHPT is the predominant form of disease in many developing countries (1). Pancreatitis is known to be associated with primary hyperparathyroidism (PHPT) ( 2,3). However the relationship of cause and effect between the two diseases continues to be debated in the medical literature. Gallstones and alcoholism are the commonest etiological agents of pancreatitis in Sri Lanka. For this reason, calcium is not routinely measured in all patients first presenting with acute pancreatitis. Some patients suffer from two or more attacks of pancreatitis before a diagnosis of PHPT is made. We present a patient who had repeated admissions for acute pancreatitis - five episodes over a period of five months before the final diagnosis of PHPT was made.

\section{Case report}

We report a 53 year old lady who had presented to a surgical unit with severe upper abdominal pain, vomiting and fever of one day duration. Acute cholecystitis was excluded and after proper evaluation a diagnosis of pancreatitis was made, and the patient was managed conservatively. This initial episode of pancreatitis was complicated with ARDS and acute renal failure. In the past she had undergone ureterolithotomy for bilateral urteric calculi. Within the next 3 months she was admitted with further four episodes of recurrent pancreatitis. During the first 3 episodes of pancreatitis, the serum calcium was not estimated and during the fourth episode the serum calcium was $2.41 \mathrm{mmol} / \mathrm{L}(2.2-2.6 \mathrm{mmol} / \mathrm{L})$ and serum albumin was $4.6 \mathrm{~g} / \mathrm{dL}$. After the acute episode of pancreatitis subsided, serum biochemical parameters were repeated. Repeat serum calcium was $2.8 \mathrm{mmol} / \mathrm{L}$ (2.2-2.6 $\mathrm{mmol} / \mathrm{L}$ ), phosphate $4.29 \mathrm{mg} / \mathrm{dL}$ (2.48 - 4.65), albumin 4.8 $\mathrm{g} / \mathrm{dL}$, and serum alkaline phosphatase was $284 \mathrm{U} / \mathrm{L}$. Repeated serum estimations confirmed persistent hypercalcemia and a $24 \mathrm{~h}$ urinary calcium of $8.37 \mathrm{mmol} /$ 24h (2.5-7.5). The initial suspicion of primary hyperparathyroidism was confirmed when her intact parathyroid hormone level (iPTH) level was found to be $151 \mathrm{pg} / \mathrm{ml}$ (10-69).Ultrasonography neck revealed a $7 \mathrm{~mm}$ x $10 \mathrm{~mm} \times$ $16 \mathrm{~mm}$ well defined low echogenic mass behind the lower pole of R/lobe of thyroid which was later confirmed with CT scan. Right inferior parathyroid adenoma was localized on a radionuclide parathyroid scan (99 mTC Sestamibi)

${ }^{1}$ Senior Registrar in Endocrinology, National Hospital of Sri Lanka, Colombo. ${ }^{2}$ Consultant Endocrinologist, ${ }^{3}$ Consultant Surgeon, Professorial Unit, Colombo South Teaching Hospital, Sri Lanka. 
(Figure 1). She underwent traditional four gland exploration and parathyroid adenoma was removed (Figure 1). Intraoperative PTH was not performed due to unavailability and PTH postoperatively was 11.10 pg/ml (10-69). She developed hypocalcemic symptoms on 2nd post- operative day and was started on calcium replacement. Her serum calcium was monitored and gradually calcium replacement was reduced and omitted 6 weeks post operatively. Presently the patient is asymptomatic and her latest serum calcium was $2.3 \mathrm{mmol} / \mathrm{L}$ (2.2-2.6).
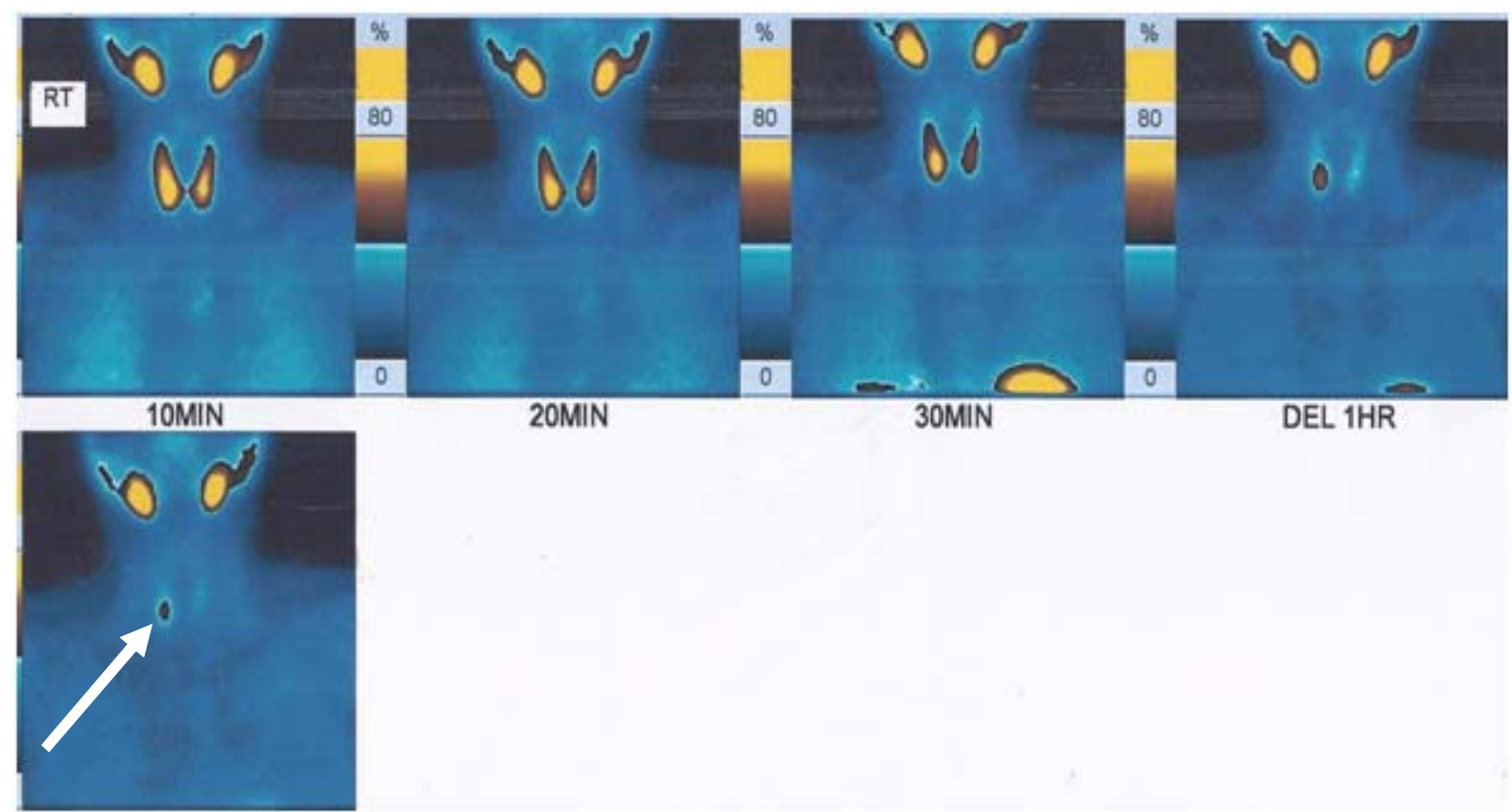

Figure 1. Sestamibi scan showing right parathyroid adenoma.

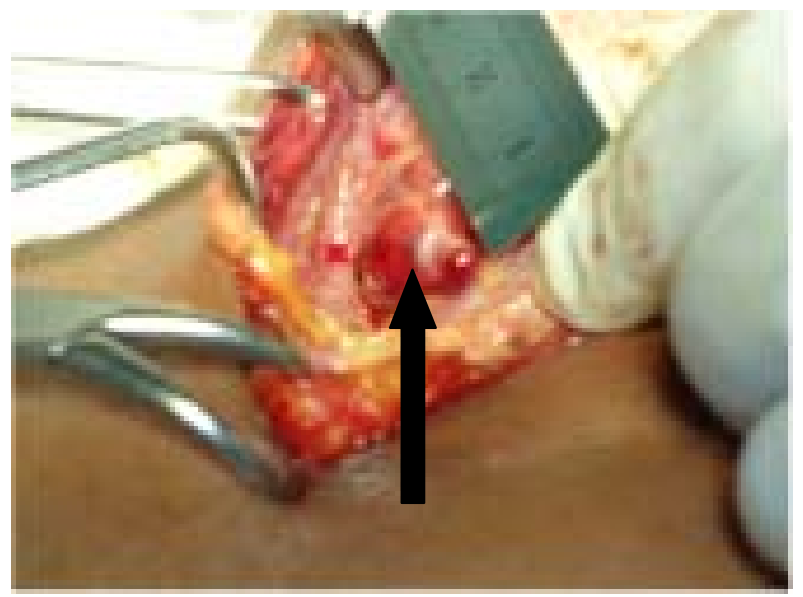

Figure 2. Black arrow showing the parathyroid adenoma intraoperatively.

\section{Discussion}

The association between pancreatitis and primary hyperparathyroidism was probably first described by Erdheim in 1903 when he reported a patient with multiple endocrine tumours including a parathyroid adenoma, who was found at postmortem to have a necrotic pancreas (2). Since then, there have been many individual cases and case series reported periodically in the belief that the concomitance of hyperparathyroidism and pancreatitis is more than coincidental and that recurrent pancreatitis can be cured by treating the cause of primary hyperparathyroidism $(3,4)$.

The first largest series was reported by Mixter et al. They reported eleven cases of pancreatitis out of 155 cases of hyperparathyroidism in Massachusetts General Hospital between 1950 and 1962. Mixter estimated that pancreatitis occurred in between $7 \%$ and $10 \%$ of patients with hyperparathyroidism (5). This observation was later challenged by Bess and colleagues from Mayo clinic in 1980. They reviewed 1153 patients and showed the coexistence or history of pancreatitis was found only in $1.5 \%$ of 
patients similar to the frequency of acute pancreatitis observed in the matched control group and suggested that the association may be casual rather than causal (6). Much later publications dealing with parathyroid have shown increased association of pancreatitis from 3.2 to $5.6 \%(7,8,9)$. These publications criticize the report by Bess and coworkers and point out that the reason for the low incidence is that most of these patients present in the asymptomatic stage with slight hypercalcaemia and are submitted to parathyroid surgery before they have a chance to develop pancreatitis. Studies from India report an incidence between $6.8 \%-12 \%(7,8)$. The possible explanation for the high incidence of pancreatitis in our part of the world could be explained by the fact that most of our patients present with symptomatic disease and they have relatively higher calcium values than in the West.

The presentation of PHPT as pancreatic disease occurs at a younger age compared with the median age of presentation in isolated PHPT. The sex distribution also shows a male preponderance. This is in contrast to the female preponderance that is observed in PHPT without associated pancreatic disease. In one of the largest series of patients with PHPT and pancreatic disease from France the mean age in patients with pancreatitis and PHPT was 52.5 compared to 55.5 in patients with PHPT without pancreatic disease. The male: female ratios were 1.1:1 and 0.34 : 1 , respectively (9).

The presentation of pancreatic disease in PHPT falls into four important classes: $(7,10)$

- PHPT presenting as acute pancreatitis

- PHPT presenting as acute recurrent pancreatitis with no evidence of chronic pancreatitis

- PHPT presenting as chronic pancreatitis with or without pancreatic calcification

- PHPT complicated by acute pancreatitis in the postoperative period

A review of the literature on the clinical presentation of various case series revealed that PHPT presenting as acute pancreatitis was the most common, occurring in over $44 \%$ of patients, underlining the need to check calcium values in all patients presenting with the first episode of pancreatitis. In over one-third of the patients the disease presented as chronic pancreatitis. Parathyroid surgery may not reverse the pancreatic pathology in cases with chronic pancreatitis. However, it is still important to check serum calcium levels in these patients as early parathyroid surgery would prevent associated renal and bone disease $(7,9,10)$.

There are three mechanisms described for the development of acute pancreatitis in patients with PHPT. Kelly (1968), on the basis of his experimental study on rats, showed that persistent hypercalcmia from PHPT leads to de novo activation of trypsinogen to trypsin, resulting in autodigestion of the pancreas and subsequent pancreatitis (11). In various studies the mean calcium values were significantly higher among patients with PHPT and pancreatic disease compared with patients with PHPT without pancreatic involvement. This suggests that the mechanism of development of pancreatic disease in PHPT is better correlated to the hypercalcaemia rather than the increased PTH. This could be another reason why the incidence is low in the West because as emphasized their main presentation is asymptomatic disease with mild elevation in serum calcium levels $(7,12)$.

Another mechanism for the development of acute pancreatitis in PHPT is that hypercalcemia leads to formation of pancreatic calculi, ductal obstruction, and subsequent attacks of acute or chronic pancreatitis $(7,8,12)$. Although most patients with primary hyperparathyroidism had elevated serum calcium not all of them developed pancreatitis. This controversial result suggests that additional disease-modifying factors may be important for the development of pancreatitis in PHPT patients. Mutations in the serine protease inhibitor Kazal type I (SPINK1) gene and cystic fibrosis transmembrane conductance regulator (CFTR) gene are proven to be associated with idiopathic chronic pancreatitis (ICP), tropical pancreatitis, and alcoholic pancreatitis .Therefore studies to explore whether these mutations in genes also coexisted in patients with PHPT concluded that they may facilitate the development of pancreatitis in situations of intense hypercalcemia (13). Studies are ongoing on other genes such as CASR (calcium-sensing receptor), but their role in this process is not clear yet (14).

\section{Conclusion}

Recent studies conclude that there is a causal association between the pancreatic disease and PHPT. The exact cellular mechanism by which hypercalcaemia causes pancreatic injury in PHPT remains to be elucidated. Until more information is available, it would be prudent to check serum calcium in all patients presenting with unexplained pancreatic disease.

\section{References}

1. Bilezikian JP, Silverberg SJ. Clinical practice. Asymptomatic primary hyperparathyroidism. New England Journal of Medicine 2004; 350: 1746-51.

2. Yönetçi Nadir, Oruç Nevin, Yımaz Mustafa, Acute pancreatitis due to primary hyperparathyroidism: A case report. The Turkish Journal of Gastroenterology 2000; 11: 4-5.

3. Smith FB, Cooke RT. Acute fatal hyperparathyroidism. Lancet 1940; 2: 560-61. 
4. Cope O, et al. Pancreatitis, a diagnostic clue to hyperparathyroidism. Annals of Surgery 1957; 145: 857-63.

5. Mixter, CG, Keynes M, Cope. Further experience with pancreatitis as a diagnostic clue to hyperparathyroidism. New England Journal of Medicine 1962; 266: 265.

6. Bess MA. Edis AJ, van Heerden JA. Hyperparathyroidism and Pancreatitis.Chance or a Causal Association? JAMA 1980; 243: 246-7.

7. Jacob JJ et al. Does hyperparathyroidism cause pancreatitis? A South Indian experience and a review of published work. ANZ Journal of Surgery 2006; 76: 740-4.

8. Agarwal A, George RK, Gupta SK, et al. Pancreatitis in patients with primary hyperparathyroidism. Indian Journal of Gastroenterology 2003; 22: 224-5.

9. Carnaille B, Oudar C, Pattou F, Combemale F, Rocha J, Proye C. Pancreatitis and primary hyperparathyroidism: forty cases. ANZ Journal of Surgery 1998; 68: 117-19.
10. Pyrah LN, Hodgkinson A, Anderson CK. Primary hyperpara-thyroidism. British Journal of Surgery 1966; 53: $245-316$

11. Kelly TR. Relationship of hyperparathyroidism to pancreatitis. Archives of Surgery 1968; 97: 267.

12. Raiz A, Mathew MV, et al. Primary Hyperparathyroidism presenting as recurrent acute pancreatitis: A case report and review of literature. Indian Journal of Endocrinology and Metabolism 2011; 15: 54-56.

13. Felderbauer $P$ et al. Pancreatitis Risk in Primary Hyperparathyroidism:Relation to Mutations in the SPINK1 Trypsin Inhibitor (N34S) and the Cystic Fibrosis Gene. American Journal of Gastroenterology 2008; 103: 368-74.

14. Felderbauer P, Karakas E, Fendrich V, et al. Pancreatitis in primary hyperparathyroidism related hypercalcaemia is not associated with mutations in the CASR gene. Exp Clin Endocrinol Diabetes 2007; 115: 527-9. 